\title{
Desafios associados à esclerose lateral amiotrófica: relato de caso clínico
}

\author{
Challenges associated amyrotrophic lateral sclerosis: clinical case report
}

Desafíos asociados a la esclerosis lateral amirotrofica: informe clinico de caso

Tamara Simão Bosse $^{1 *}$, Lara Ferreira Baptista ${ }^{1}$, Gustavo Gavilan Alves da Silva Junior ${ }^{1}$, Rebeca Coêlho Linhares ${ }^{1}$, Élida Maria Moura de Paulo Portela ${ }^{2}$, Isadora Maria de Almeida Morais ${ }^{3}$, Luana Cristina Farias Castro ${ }^{1}$, Luan Kelves Miranda de Souza'1.

\section{RESUMO}

Objetivo: Relatar o caso e descrever os desafios associados à Esclerose Lateral Amiotrófica, analisando seus efeitos. Detalhamentos de Caso: A Esclerose Lateral Amiotrófica (ELA) é uma doença neurodegenerativa progressiva, idiopática e fatal que afeta os neurônios motores na medula espinal, tronco cerebral e córtex motor. Na busca de entender os desafios enfrentados por uma paciente portadora de ELA, foi avaliado as terapias adjuvantes no retardo do avanço da doença, além de que, nesse estudo foi possível perceber que a implantação do marca-passo diafragmático reforçou a musculatura, na qual obteve mais força para realizar movimentos respiratórios, que a redução proporcional da Capacidade Vital Forçada (CVF) (46\%) e do Volume Expiratório Forçado no primeiro minuto (VEF1) (50\%) se tornou grave e que os transplantes de células estaminais hematopoiéticas obtiveram uma melhora na força por 6 meses e na fala. Considerações Finais: Porém mais estudos e pesquisas devem ser realizados investigando melhor os mecanismos de ação das terapias coadjuvantes no tratamento da ELA com a combinação de farmacoterapia para aumentar a sobrevivência e otimizar sua qualidade de vida, além de ajudar na busca de novos tratamentos.

Palavras-chave: Esclerose lateral amiotrófica, Neurônios motores, Terapêuticas.

\begin{abstract}
Objective: To report the case and describe the challenges associated with Amyotrophic Lateral Sclerosis, analyzing its effects. Case Details: Amyotrophic Lateral Sclerosis (ALS) is a progressive, idiopathic and fatal neurodegenerative disease that affects motor neurons in the spinal cord, brain stem and motor cortex. In an attempt to understand the challenges faced by a patient with ALS, adjuvant therapies were evaluated to delay the progress of the disease, in addition to this study showing that the implantation of the diaphragmatic pacemaker strengthened the musculature, in which she obtained more force to perform respiratory movements, that the proportional reduction of Forced Vital Capacity (FVC) (46\%) and Forced Expiratory Volume in the first minute (FEV1) $(50 \%)$ became severe and that hematopoietic stem cell transplants obtained a improvement in strength for 6 months and in speech. Final Considerations: However, more studies and research should be carried out to better investigate the mechanisms of action of supporting therapies in the treatment of ALS with the combination of pharmacotherapy to increase survival and optimize their quality of life, in addition to helping in the search for new treatments.
\end{abstract}

Keywords: Amyotrophic lateral sclerosis, Motor neurons, Therapies.

\footnotetext{
${ }^{1}$ Instituto de Educação Superior do Vale do Parnaíba (IESVAP), Parnaíba-PI. *E-mail: tamarasimaobosse@hotmail.com

2 Centro Universitário UNINOVAFAPI (UNINOVAFAPI), Teresina-PI.

3 Universidade Federal do Piauí (UFPI), Parnaíba-PI.
} 


\section{RESUMEN}

Objetivo: Informar el caso y describir los desafíos asociados con la esclerosis lateral amiotrófica, analizando sus efectos. Detalles del caso: La esclerosis lateral amiotrófica (ELA) es una enfermedad neurodegenerativa progresiva, idiopática y mortal que afecta a las neuronas motoras de la médula espinal, el tronco encefálico y la corteza motora. En un intento por comprender los desafíos que enfrenta un paciente con ELA, se evaluaron las terapias adyuvantes para retrasar el progreso de la enfermedad, además de este estudio que muestra que la implantación del marcapasos diafragmático fortaleció la musculatura, en la que obtuvo mayor fuerza para realizar movimientos respiratorios, que la reducción proporcional de la capacidad vital forzada (FVC) (46\%) y el volumen espiratorio forzado en el primer minuto (FEV1) $(50 \%)$ se volvió severa y que los trasplantes de células madre hematopoyéticas obtuvieron un mejora en la fuerza durante 6 meses y en el habla. Consideraciones finales: Sin embargo, se deben realizar más estudios e investigaciones para investigar mejor los mecanismos de acción de las terapias de apoyo en el tratamiento de la ELA con la combinación de farmacoterapia para aumentar la supervivencia y optimizar su calidad de vida, además de ayudar en la búsqueda de nuevos tratamientos.

Palabras clave: Esclerosis lateral amiotrófica, Neuronas motoras, Terapias.

\section{INTRODUÇÃO}

A Esclerose Lateral Amiotrófica (ELA) é uma doença neurodegenerativa progressiva, idiopática e fatal que afeta os neurônios motores na medula espinal, tronco cerebral e córtex motor. Trata-se de uma patologia de difícil diagnóstico precoce e terapêutica dependente de uma atenção multidisciplinar, tendo prognóstico positivo em relação aos tratamentos paliativos (FACCHINETTI LD, ORSINI M e LIMA MASD, 2009; BENSTEAD T, JACKSON-TARLTON C e LEDDIN D, 2016; HERNÁNDEZ RG, et al., 2017).

Um conjunto de variáveis tem sido investigado como possíveis fatores de risco para ELA, considerando desde causas genéticas, 5 a 10\% com padrão de herança autossômica dominante, até fatores ambientais, químicos e físicos. Entretanto cerca de $90 \%$ são considerados esporádicos e com etiologia desconhecida (LEITE NETO L. e CONSTANTINI, AC, 2017; ORSINI M, et al., 2017; SIQUEIRA SC, et al., 2017; SILVA L.P, et al., 2018).

A ELA caracteriza-se por uma degeneração progressiva de neurônios motores inferiores e superiores, resultando em atrofia muscular, perda de peso, paralisia bulbária progressiva, fasciculação, fraqueza e espasticidade. Com o avanço da doença os indivíduos passam a ter maior comprometimento funcional, com declínio da capacidade na realização de atividades básicas da vida diária, tornando-os dependentes dos cuidados de terceiros (OLIVEIRA ASB e PEREIRA RDB, 2009; LEITE NETO L. e CONSTANTINI, AC, 2017; SILVA L.P, et al., 2018).

Acredita-se que a incidência da ELA seja a mesma em todo o mundo, porém, a maior parte destes estudos é proveniente da América do Norte e Europa. No mundo a incidência por ano é de 2/100.000 e a prevalência é de 3 - 8 casos por 100.000 habitantes. Entretanto, estudos isolados mostram que existem variações. A maioria desses indivíduos encontra-se entre 40 e 70 anos. E a sobrevida da metade desses pacientes afetados vivem pelo menos 3 anos ou mais após o diagnóstico. Os que sobrevivem cinco anos ou mais é em torno de 20\%, e apenas 10\% sobrevivem mais de 10 anos (PEREIRA RDB, 2006; SILVA LP, et al., 2018).

No Brasil, até o momento, não foram feitos estudos relevantes sobre a prevalência e incidência de ELA em uma grande população. Conhecer esses dados é importante para auxiliar no diagnóstico precoce e cuidado paliativo, sendo esse definido como aquele prestado a fim de promover a qualidade de vida de indivíduos que sofrem de doenças que ameaçam a vida, por meio da prevenção e do alívio do sofrimento físico, psicossocial e espiritual, bem como ajudar na busca por novos tratamentos e até mesmo a cura da doença (LUCHESI KF e SILVEIRA IC, 2018).

Este estudo teve com objetivo relatar e descrever os desafios associados à Esclerose Lateral Amiotrófica (ELA), bem como analisar os seus efeitos em um relato de caso. 


\section{DETALHAMENTO DO CASO}

Paciente do sexo feminino, 28 anos, solteira, estudante de enfermagem, procedente de Balneário GaivotaSC iniciou quadro de algia em ombro direito no ano de 2009. Em 2010, apresentou parestesia no quinto quirodáctilo direito, câimbras em membro inferior direito, bem como quedas frequentes da própria altura.

Dois anos após o surgimento dos primeiros sinais e sintomas, as manifestações da doença começaram a ficar mais evidentes, com o surgimento de marcha alterada em membro inferior direito, fasciculações e perda da força no indicador e polegar direito a pequenos esforços como consta na Quadro 1.

Quadro 1 - Evolução da Esclerose Lateral Amiotrófica da paciente estudada. Balneário Gaivota, 2018.

\begin{tabular}{|c|c|}
\hline Período & Sinais / sintomas \\
\hline Novembro 2009 & Algia no ombro direito \\
\hline Fevereiro 2010 & Parestesia no quinto quirodáctilo direito \\
\hline Junho 2010 & Câimbras membro inferir direito \\
\hline Novembro 2010 & Quedas com sapato de salto \\
\hline Janeiro 2011 & Marcha alterada no membro \\
\hline Fevereiro 2011 & Fasciculações \\
\hline Maio de 2011 & $\begin{array}{l}\text { Indicador e polegar direito perdem força a } \\
\text { pequenos esforços }\end{array}$ \\
\hline Junho 2011 & DIAGNÓSTICO ELA \\
\hline Fevereiro 2012 & $\begin{array}{l}\text { Transplante células tronco na Alemanha (melhora } \\
\text { da força por seis meses) }\end{array}$ \\
\hline Até 2013 & Ainda dirigia \\
\hline 2013 & $\begin{array}{l}\text { Quedas e fraqueza evidente de membros } \\
\text { superiores }\end{array}$ \\
\hline $17-12-2013$ & Cirurgia marca-passo diafragmático \\
\hline Abril 2014 & Parou de se alimentar com as próprias mãos \\
\hline Maio/Junho 2014 & $\begin{array}{l}\text { Transplante de células tronco no México (melhora } \\
\qquad \text { na fala) }\end{array}$ \\
\hline Janeiro 2015 & $\begin{array}{l}\text { Fisioterapia motora respiratória e fonoaudiologia } \\
\text { domiciliar }\end{array}$ \\
\hline 14-09-2016 & Cirurgia gastrostomia \\
\hline Fevereiro 2017 & $\begin{array}{l}\text { Pressão Positiva em dois Níveis das Vias Aéreas } \\
\text { (BIPAP) (uso noturno março em diante) }\end{array}$ \\
\hline Agosto 2017 & $\begin{array}{l}\text { Paresia de pescoço e tronco com força muscular } \\
\text { diminuídas }\end{array}$ \\
\hline Janeiro 2018 & Algia joelho esquerdo \\
\hline Fevereiro 2018 & Apendicectomia \\
\hline
\end{tabular}

Fonte: Bosse TS, et al., 2018.

A progressão dos acometimentos fez com que a paciente procurasse o serviço de saúde especializado. Foram realizados exames laboratoriais, com o intuito de eliminar algumas suspeitas patológicas, e o exame de eletroneuromiografia, para pesquisar neuropatias e ELA. Em junho de 2011 foi confirmado, através da eletroneuromiografia, o diagnóstico de Esclerose Lateral Amiotrófica da paciente.

Durante o tratamento, percebe-se no gráfico da Capacidade Vital Forçada (CVF) (Figura 1), ao longo dos seis anos, a decadência da sua capacidade respiratória em função da fraqueza dos músculos que envolvem o sistema respiratório. 
Figura 1 - Porcentagem da CVF de acordo com os anos.

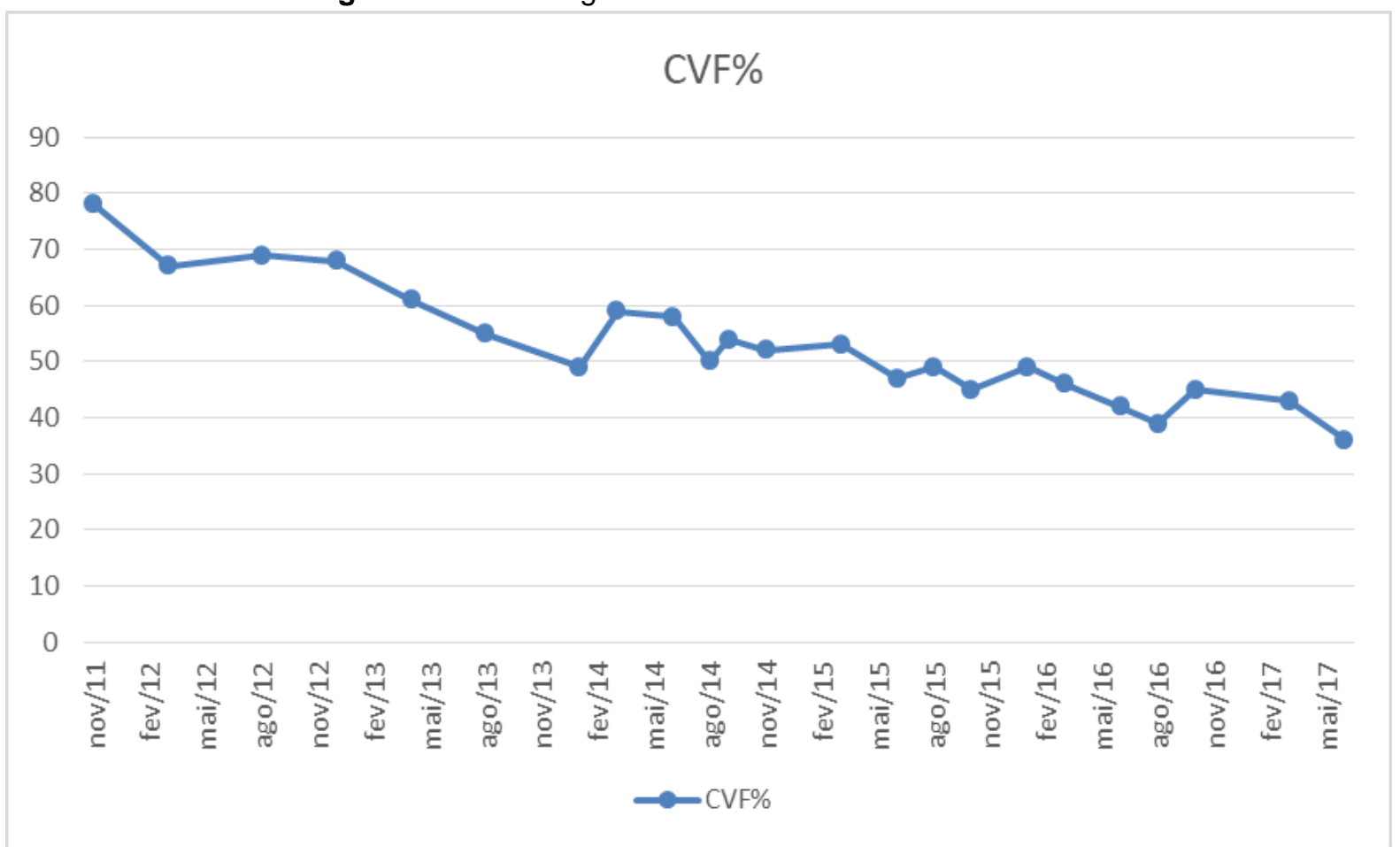

Fonte: Bosse TS, et al.,2018.

Na Figura 2, pode-se observar uma redução proporcional na capacidade da função vital e do volume expiratório no primeiro minuto, durante os seis anos. No decorrer desses anos visualiza-se algumas melhoras sendo essas relacionadas aos tratamentos realizados pela paciente, porém o gráfico mostra um decréscimo nessas duas funções.

Figura 2 - Comparação de gráficos CVF e VEF1 de acordo com os anos (2011-2017)

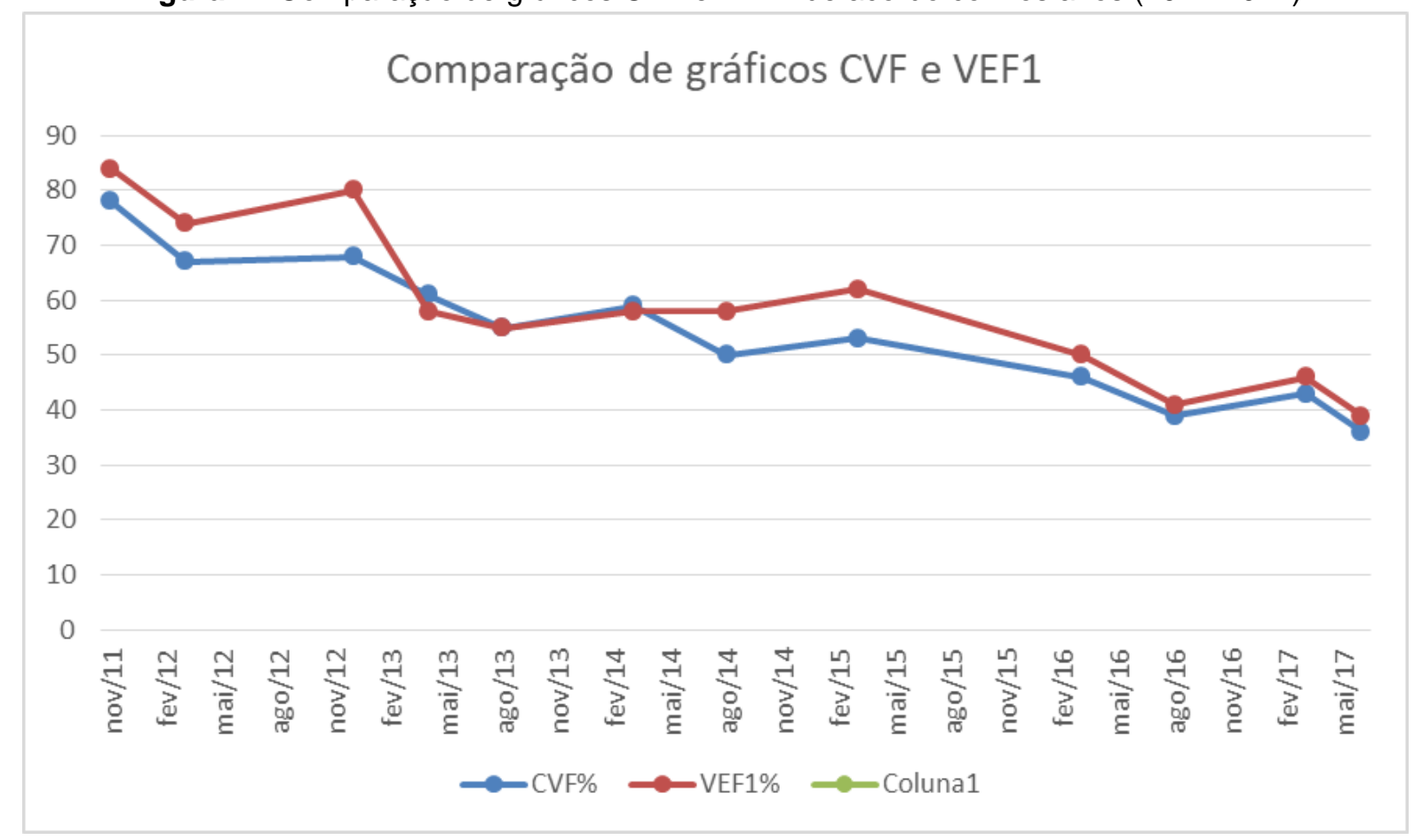

Fonte: Bosse TS, et al.,2018. 
A paciente submeteu-se à realização de dois transplantes de células estaminais hematopoiéticas realizados na Alemanha em fevereiro de 2012 e no México em maio/junho de 2014, obtendo como resposta melhora na força, por seis meses, e na fala.

\section{DISCUSSÃO}

A partir dos resultados obtidos nesse estudo foi possível observar que para confirmar o diagnóstico de Esclerose Lateral Amiotrófica não há um exame específico. Entretanto existe exames que auxiliam na confirmação como a eletroneuromiografia (EMG) que é um exame utilizado a partir da análise da transmissão das junções neuromusculares através de eletrodos, verificando seu funcionamento ou suas falhas (LIU M, et al., 2016).

Entretanto, seus sinais devem ser bem interpretados e classificados a fim de diferenciar ELA (neuronais) de miopatias (distúrbios musculares), pois o tratamento dessas doenças é diferente (ARTAMEEYANANT P, et al., 2016). O diagnóstico por ELA se dá pela associação de sinais de comprometimento neuromotor crônico ou progressivo.

O exame de EMG engloba toda a zona da coluna vertical, incluindo, a língua, região abdominal, torácica e paravertebral, tendo também uma procura de deficiência motora nas localidades distais e proximais dos membros inferiores e superiores. São necessárias realizações repetidas desse exame para que evite falsopositivos (CHIEIA MA, 2010). As complicações respiratórias tendem a ficar cada vez mais comprometidas, uma vez que a fraqueza muscular progressiva afeta músculos de fundamental importância, sendo ela responsável por mais de $85 \%$ das mortes. A respiração destes pacientes é classificada como padrão restritivo, visto que afeta capacidade vital forçada (CVF) e a ventilação-perfusão (TILANUS TBM, 2017).

$\mathrm{Na}$ CVF ao longo dos seis anos, percebe-se a decadência da sua capacidade respiratória em função da fraqueza dos músculos que envolvem o sistema respiratório. Observa-se um leve aumento entre janeiro e março de 2014 em que os exames 01-2014 (CVF 49\%) e 03-2014 (CVF 59\%) mostraram a sua melhora, pois com a implantação do marca-passo diafragmático, houve um reforço muscular ajudando a obter mais força ao realizar os movimentos respiratórios.

O marca-passo diafragmático (DPS) foi outra medida a ser adotada pela portadora da doença a partir de dezembro de 2013, visto que a diminuição da capacidade vital pode ser controlada através de estimulações de baixa frequência do músculo diafragmático, pois a insuficiência ventilatória está relacionada à dependência da falha diafragmática. Assim, a interferência por estimulação elétrica ajuda na qualidade do sono, promovendo também um maior desenvolvimento de fibras musculares tipo 1(GONZALEZ-BERMEJO J, 2011; TERTEMIZ, 2017).

A ventilação por pressão positiva em dois níveis das vias aéreas (BIPAP) é outra opção de ventilação nãoinvasiva (VNI), sendo bastante útil para os cuidados paliativos e lidar com a hipoventilação cada vez maior com a decadência da função do diafragma (SELLNER-POGÁNY T e LAHRMANN H, 2009). Sendo ele utilizado pela paciente desde fevereiro de 2017 com efetivação. Assim, a utilização de ventilação não-invasiva pode garantir uma melhor qualidade de vida destes pacientes, reduzindo a dispneia, melhorando o sono e a hipoventilação alveolar com auxílio de exames da função pulmonar que avaliem capacidades vitais lentas (CVL) e forçadas (CVF) nos quais são os testes mais usados na ELA (PINTO S e CARVALHO M, 2017).

Portadores de esclerose lateral amiotrófica devem ser acompanhados por equipes de cuidado trimestralmente antes de serem encaminhados para um serviço de ventilação domiciliar. Dessa forma, o uso da espirometria, um dos principais exames para a avaliação da capacidade pulmonar, é de fundamental importância utilizando como referências a capacidade vital forçada menor que $70 \%$, hipercapnia diurna e $\mathrm{PCO}_{2}$ maior que 45mmHg para dar início ao uso da NIV (TILANUS TBM, et al., 2017).

Até o ano de 2013, todos os critérios da Sociedade Torácica Americana e da Sociedade Respiratória Europeia (ATS/ERS 2005) foram cumpridos dos melhores testes forçados. A partir de 03-2014, nos exames constaram que a CVF (59\%) teve uma redução moderada, limitação moderada ao fluxo aéreo e dificuldade para a realização de manobras, provavelmente relacionada à patologia de base, assim números insuficientes 
de testes forçados bons antes e após o uso de broncodilatadores, resultando na baixa da capacidade muscular pulmonar e dificuldade na realização de manobras, sugerindo distúrbio ventilatório combinado moderado. Em 08-2014 foi obtido uma redução moderada tanto da CVF (50\%) quanto da VEF1 (58\%), além de uma ausência de variação significativa com broncodilatador no teste. Até que em 03-2015, onde a CVF é de 53\%, observa-se uma ausência de variação significativa com broncodilatadores no teste. Em 03-2016 a redução proporcional da CVF (46\%) e do VEF1 (50\%) se tornou grave como apresentado nas figuras 2 e 3.

De acordo com a figura 1, a partir do ano de 2015 em que começou o auxílio fisioterápico e fonoaudiólogo, houve uma leve melhora entre 06-2015 (47\%) a 08-2015 (CVF 49\%), comparando-se aos exames 08-2012 (CVF 69\%) a 01-2014 (CVF 49\%) os quais houve uma perda significativa de $20 \%$ da CVF, visto que a sua queda não foi tão brusca quanto antes, ressaltando a assistência multiprofissional.

Com isso, a atuação do fonoaudiólogo é essencial, pois quando o paciente inicia o tratamento no estágio precoce da doença faz com que ele desenvolva mecanismos musculares adaptativos e diminua o risco de aspiração traqueal, mesmo com graves alterações musculares. A reabilitação mantém uma fala compreensível, usando exercícios de coordenação fono-respiratória e mobilidade labial e lingual, porém não há evidências de que esse exercício melhore a função de deglutição, mas auxilia nas diferentes fases da mesma melhorando o controle oral do bolo alimentar e utilizando técnicas facilitadoras na deglutição segura, como incluindo o tempo prolongado de mastigação, a inclinação da cabeça e a dobra do queixo (PONTES RT, et al., 2010).

Outra área que tem um papel importante no tratamento de pacientes com ELA é a fisioterapia, pois com a progressão da doença surgem muitos problemas e esses podem ser tratados utilizando-se métodos e técnicas fisioterapêuticas. Os estudos acerca do papel do exercício resistido respiratório em pacientes com ELA são poucos, porém os resultados dizem que exercícios de resistência moderados são benéficos e ajudam na manutenção da função (LINDER JUNIOR E, 2013). Já os alongamentos, ou exercícios que melhoram a flexibilidade, podem manter a extensibilidade dos músculos, tecidos moles e prevenir as alterações musculoesqueléticas causadas pela progressão da doença, tais como: encurtamento muscular, contraturas e má postura que ocorrem devido à perda progressiva da força muscular e desequilíbrio entre os grupos musculares agonistas e antagonistas.

A paciente submeteu à realização de dois transplantes de células estaminais hematopoiéticas realizados na Alemanha em fevereiro de 2012 e no México em maio/junho de 2014. Ambos os transplantes utilizavam células autólogas coletadas no sangue periférico posterior à estimulação de G-CSF. Após isso, as células mãe são injetadas através de punção lombar na medula espinhal. Ao término, a paciente é convidada a deitarse a fim de evitar cefaleia, como previsto nos efeitos colaterais do procedimento, este, que ocorrerá três vezes ao ano, além de possuir acompanhamento médico mensal.

Os procedimentos de transplantes visaram e promoveram à paciente aumento da sobrevida em três meses, diminuir a necessidade de ventilação mecânica com melhora relativa em 10 a 15\%, mas não promovia uma cura efetiva da doença. O transplante realizado na Alemanha permitiu uma melhora na força por seis meses, enquanto o do México obteve melhora na fala.

A terapia com células-tronco é um dos tratamentos mais promissores realizados nos ensaios pré-clínicos e clínicos para ELA. As discussões sobre as propriedades terapêuticas destas células são baseadas na capacidade de se diferenciar em linhagens neuronais múltiplas a partir de diferentes tipos e variedades dessas. Alguns dados desses ensaios iniciais indicam uma redução na taxa de progressão da doença, o que sugere uma eficácia favorável nesse tipo de tratamento (LIMA SR e GOMES KB, 2010).

A sobrevivência das pessoas que frequentam uma clínica especializada no tratamento para ELA melhorou ao longo de um período de treze anos a sobrevida desses pacientes (MARTIN S, et al., 2017). Mostrando que este efeito não é totalmente explicado pelas melhorias nos tratamentos disponíveis durante o tempo de permanência, mas sim apoia a ideia de que o cuidado multidisciplinar integrado fornece benefícios, mostra evidências de que a integração de cuidados paliativos, respiratórios e terapeutas na esclerose lateral amiotrófica podem ser observados na sobrevivência desses indivíduos, melhorando a qualidade de vida desses pacientes (MAJMUDAR S, et al., 2014). 
As terapias adjuvantes no paciente com ELA, como a ventilação não-invasiva, marca-passo diafragmático, fonoaudiologia e fisioterapia, têm como principal objetivo maximizar a sobrevida, trabalhando a força e função dos órgãos afetados. A combinação de farmacoterapia e outras intervenções terapêuticas podem aumentar a expectativa de vida, ajudar os pacientes a fortalecer os músculos, melhorar sua capacidade de realizar atividades de vida diária e otimizar sua qualidade de vida. Porém mais estudos e pesquisas devem ser realizados investigando melhor os mecanismos de ação das terapias coadjuvantes no tratamento da ELA com a combinação de farmacoterapia, além de ajudar na busca de novos tratamentos e até mesmo a cura da doença.

\section{REFERÊNCIAS}

1. ARTAMEEYANANT $P$, et al. An EMG-based feature extraction method using a normalized weight vertical visibility algorithm for myopathy and neuropathy detection. Springerplus, 2016; v. 5, n. 1, 1-26.

2. BENSTEAD T, et al. Nutrition with Gastrostomy Feeding Tubes for Amyotrophic Lateral Sclerosis in Canada. Canadian Journal of Neurological Sciences / Journal Canadien des Sciences Neurologiques, 2016; v. 43, n. 06, 796-800.

3. CHIEIA MA, et al. Amyotrophic lateral sclerosis: considerations on diagnostic criteria. Arquivos de Neuropsiquiatria,2010 v. 68 , n. $6,837-842$.

4. FACCHINETTI LD, et al. Os riscos do exercício excessivo na Esclerose Lateral Amiotrófica: atualização da literatura. Revista Brasileira de Neurologia, 2009; v. 45, n. 3, 33-38.

5. GONZALEZ-BERMEJO J, et al. Diaphragm pacing improves sleep in patients with amyotrophic lateral sclerosis. Amyotrophic Lateral Sclerosis, 2011; v. 13, n. 1, 44-54.

6. HERNÁNDEZ RG, et al. Extraocular Motor System Exhibits a Higher Expression of Neurotrophins When Compared with Other Brainstem Motor Systems. Frontiers In Neuroscience, 2017; v. 11, 1-12.

7. LEITE NETO L, CONSTANTINI AC. Disartria e qualidade de vida em pacientes com esclerose lateral amiotrófica. Revista Cefac, 2017; v. 19, n. 5, 664-673.

8. LIMA SR, GOMES KB. Esclerose lateral amiotrófica e o tratamento com células-tronco. Rev Bras Clin Med., 2010; V. 6, n. $8,531-537$.

9. LINDEN JUNIOR, E. Abordagem Fisioterapêutica na Esclerose Lateral Amiotrófica: Artigo de Atualização. Rev Neurocienc, 2013; v. 2, n. 21, 313-318.

10. LIU M, et al. Quantitating Changes in Jitter and Spike Number Using Concentric Needle Electrodes in Amyotrophic Lateral Sclerosis Patients. Chinese Medical Journal, 2016; v. 129, n. 9, 1036-1040.

11. LUCHESI KF, SILVEIRA IC. Cuidados paliativos, esclerose lateral amiotrófica e deglutição: estudo de caso. Codas, 2018; v. 5, n. 30, 1-6.

12. MAJMUDAR S, et al. Rehabilitation in amyotrophic lateral sclerosis: Why it matters. Muscle \& Nerve, 2014; v. 50, n. 1, 4-13.

13. MARTIN S, et al. The benefit of evolving multidisciplinary care in ALS: a diagnostic cohort survival comparison. Amyotrophic Lateral Sclerosis and Frontotemporal Degeneration,2017; 1-7.

14. OLIVEIRA ASB, PEREIRA RDB. Amyotrophic Lateral Sclerosis (ALS): Three letters that change the people's life. Arq Neuropsiquiatria, 2009; v. 3-, n. 67, 750-782.

15. ORSINI M, et al. Esclerose Lateral Amiotrófica: Novas possibilidades terapêuticas em um arcabouço fisiopatológico ainda em construção. Rev. Bras Neurol, 2017, v. 53, n. 4, 27-37.

16. PEREIRA RDB. Epidemiologia: ELA no mundo. Neurociências, 2006; v.14, n.2, 111-222.

17. PONTES RT, et al. Alterações da fonação e deglutição na Esclerose Lateral Amiotrófica: Revisão de Literatura. Revista Neurociencias, 2010; v. 18, n. 1, 69-73.

18. PINTO S, CARVALHO M. Comparison of slow and forced vital capacities on ability to predict survival in ALS. Amyotrophic Lateral Sclerosis and Frontotemporal Degeneration, 2017; 1-6.

19. SELLNER-POGÁNY T, LAHRMANN H. Die BIPAP-Maskenbeatmung in der Terminalphase der amyotrophen Lateralsklerose (ALS) - Wenn das Sterben zu Hause von einem medizinischen Gerät begleitet wird. Wiener Medizinische Wochenschrift, 2009; v. 159, n. 23-24, 604-607.

20. SILVA LP, et al. Esclerose lateral amiotrófica: descrição de aspectos clínicos e funcionais de uma série de casos numa região de saúde do nordeste do Brasil. Journal of Health \& Biological Sciences, 2018; v. 6, n. 3, 293-298.

21. SIQUEIRA SC, et al. Qualidade de vida de pacientes com Esclerose Lateral Amiotrófica. Rev Rene, 2017; v. 1, n. 18,139-146.

22. TERTEMIZ K, et al. Preoperative parameters and their prognostic value in amyotrophic lateral sclerosis patients undergoing implantation of a diaphragm pacing stimulation system. Annals of Indian Academy of Neurology, 2017; v. 20 , n. 1, 51-54.

23. TILANUS TBM, et al. The predictive value of respiratory function tests for non-invasive ventilation in amyotrophic lateral sclerosis. Respiratory Research, 2017; v. 18, n. 1, 1-10.

24. VRIJSEN B, et al. Non-invasive ventilation in amyotrophic lateral sclerosis. Amyotrophic Lateral Sclerosis and Frontotemporal Degeneration, 2013; v. 14, n. 2, 85-95. 